\title{
SAND2000-0262J
}

\section{PHOTOLUMINESCENCE-LINEWIDTH-DERIVED EXCITON MASS FOR InGaAsN ALLOYS}

\author{
E. D. Jones, A. A. Allerman, S. R. Kurtz. and N. A. Modine
} Sandia National Laboratories, Albuquerque, NM

K. K. Bajaj

Emory University, Atlanta, GA

S. T. Tozer and Xing Wei

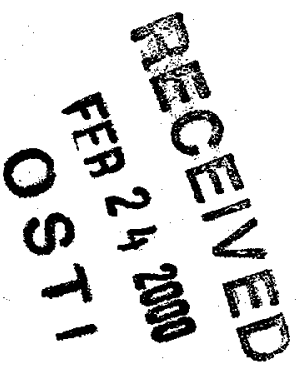

National High Magnetic Field Laboratory, Tallahassee, FL

\begin{abstract}
We report a measurement of the variation of the value of the linewidth of an excitonic transition in InGaAsN alloys ( $1 \& 2 \%$ nitrogen) as a function of hydrostatic pressure using photoluminescence spectroscopy. The samples were grown by metal-organic chemical vapor deposition and the photoluminescence measurements were performed at $4 \mathrm{~K}$. We find that the value of the excitonic linewidth increases as a function of pressure until about $100 \mathrm{kbars}$ after which it tends to saturate. This change in the excitonic linewidth is used to derive the pressure variation of the reduced mass of the exciton using a theoretical formalism which is based on the premise that the broadening of the excitonic transition is caused primarily by compositional fluctuations in a completely disordered alloy. The variation of the excitonic reduced mass thus derived is compared with that recently determined using a first-principles band structure calculation based on local density approximation.
\end{abstract}

\section{Introduction}

Since the early work of Weyers et. al. ${ }^{1,2}$ and Kondow et. al., ${ }^{3,4}$ there has been considerable interest in the study of the growth, structural, electronic, and optical properties of GaAsN and InGaAsN semiconductor alloy systems. These early reports showed that the addition of a small amount of nitrogen, e.g., about $2 \%$ in GaAsN, could cause a bandgap energy reduction approaching $0.4 \mathrm{eV}$ ! This ability to tune the bandgap energy makes it an attractive material system for opto-electronic devices operating in the 1 to $1.3 \mu \mathrm{m}$ region. Additionally, incorporating a small amount of indium ( $\sim 7 \%$ for $\left.\mathrm{GaAs}_{0.98} \mathrm{~N}_{0.02}\right)$ compensates for the strain effect of nitrogen and makes it lattice matched with GaAs, thus allowing strain free growth on readily available GaAs 
substrates. This system is thus an ideal candidate for strain-free $1.3-\mu \mathrm{m}$ lasers or multi-junction solar cells lattice matched to GaAs. ${ }^{2-6}$

Recently, Jones et al. ${ }^{7}$ have reported a first-principles calculation of the electronic band structure of this alloy system based on local density approximation. They find that the large bandgap reduction $(\sim 0.4 \mathrm{eV}$ for $2 \% \mathrm{~N}$ in lattice-matched $\operatorname{InGaAsN})$ is a result of $\Gamma-\mathrm{L}$ and $\Gamma-\mathrm{X}$ mixing and hence resulting in an energy repulsion between the $\Gamma-\mathrm{L}$ and $\Gamma-\mathrm{X}$ GaAs conduction bands. The pressure dependence of the $\Gamma$-like conduction band was discussed for the case of $2 \%$ nitrogen in InGaAsN and excellent agreement between theory and experiment was found. The exciton reduced masses and their pressure dependencies for the $2 \%$ nitrogen InGaAsN alloys were subsequently reported by Jones, et. al. using three different techniques, magnetoluminescence spectroscopy, room temperature photomodulated reflectance spectroscopy and low temperature photoluminescence (PL) as a function of the quantum well-width for lattice matched InGaAsN/ GaAs structures. ${ }^{8}$ The $4-\mathrm{K}$ ambient-pressure reduced effective mass $\mu$ was found ${ }^{8}$ to be in the range of $0.13 m_{0}-0.15 m_{0}$ where $m_{0}$ is the free electron mass. Henceforth in this paper, the free electron mass $m_{0}$ will be implicitly assumed when expressing values for the reduced effective mass $\mu$ of the exciton. The reduced mass $\mu \sim 0.13$ for $2 \%$ nitrogen in InGaAsN is about a factor of two to three times heavier than GaAs, while the pressure dependence of $\mu$ was also much greater than found for the binary GaAs system. ${ }^{8}$ The large pressure dependence for the mass was also attributed to the $\Gamma-\mathrm{L}$ and $\Gamma-\mathrm{X}$ mixing and at high pressures, where the repulsion between the $\Gamma$ like and $X$-like bands is greatest, the effect of mixing the heavier X-band mass with the lighter $\Gamma$ band mass leads to heavier, pressure dependent conduction-band masses.

In this paper we report the behavior of the full-width-at-half-maximum (FWHM) PL linewidth of an excitonic transition in InGaAsN alloys with $1 \%$ nitrogen as a function of hydrostatic pressure using PL spectroscopy. In order to provide an interpretation for this FWHM data, we need to include data from our earlier work for the $2 \%$ nitrogen InGaAsN/GaAs structure. The samples were grown on GaAs substrates by metal-organic chemical vapor phase deposition and the luminescence measurements were carried out at $4 \mathrm{~K}$. We find that the value of the excitonic linewidth $(\sigma)$ increases as a function of pressure until about $100 \mathrm{kbar}$ after which it tends to saturate. This behavior of $\sigma$ is used to derive the variation of reduced mass of an exciton $\mu$ with pres- 


\section{DISCLAIMER}

This report was prepared as an account of work sponsored by an agency of the United States Government. Neither the United States Government nor any agency thereof, nor any of their employees, make any warranty, express or implied, or assumes any legal liability or responsibility for the accuracy, completeness, or usefulness of any information, apparatus, product, or process disclosed, or represents that its use would not infringe privately owned rights. Reference herein to any specific commercial product, process, or service by trade name, trademark, manufacturer, or otherwise does not necessarily constitute or imply its endorsement, recommendation, or favoring by the United States Government or any agency thereof. The views and opinions of authors expressed herein do not necessarily state or reflect those of the United States Government or any agency thereof. 


\section{DISCLAIMER}

Portions of this document may be illegible in electronic image products. Images are produced from the best available original document. 
sure in this alloy system using a theoretical formalism of excitonic linewidth in semiconductor alloys. In this formalism it is assumed that at low temperatures the broadening of the excitonic transition in relatively good quality alloys is primarily caused by the compositional disorder expe-

rienced by the excitons. ${ }^{9}$ The extent of the excitonic charge distribution which depends on its reduced mass plays an important role in determining the value of $\sigma$. The variation of $\sigma$ with pressure thus obtained is discussed in light of the first-principles LDA bandstructure calculation of Jones et. al. ${ }^{7}$ and finally, the values for the $\sigma$-derived masses are in excellent agreement with the more traditional methods for mass determination. ${ }^{8}$

\section{Experimental}

The InGaAsN structures were grown in a vertical flow, high speed rotating disk, EMCORE GS/3200 metalorganic chemical vapor deposition reactor using trimethylindium, trimethylgallium, $100 \%$ arsine and dimethylhydrazine as the gas sources. Dimethylhydrazine was used as the nitrogen source since it has a lower disassociation temperature than ammonia and has a vapor pressure of approximately 110 torr at $18^{\circ} \mathrm{C}$. The unintentionally doped InGaAsN was nominally p-type. InGaAsN films for Hall and optical measurements were grown on semi-insulating GaAs orientated $2^{\circ}$ off $(100)$ towards $<110>$. Lattice matched $\left(\delta \mathrm{a} / \mathrm{a}<8 \times 10^{-4}\right)$ films were grown at $600^{\circ} \mathrm{C}$ and 60 torr. The growth rate was $10 \AA / \mathrm{s}$. The nominal compositions for the samples reported here $(\mathrm{N} \sim 1$ and $2 \%$ ) were determined by calibration growths of GaAsN and InGaAs along with double crystal x-ray diffraction measurements. A significant increase in photoluminescence intensity was observed from these films following a post-growth anneal. Ex-situ, postgrowth anneals were carried out in a rapid thermal anneal system under nitrogen using a sacrificial GaAs wafer in close proximity to the InGaAsN sample. A complete description of the growth techniques and other important growth/annealing parameters has been presented by Allerman et. al. ${ }^{10}$ Transmission electron microscopy measurements indicate that the samples are random and no evidence for clustering or phase separation was observed. ${ }^{11}$

The pressure was generated using a small $\mathrm{BeCu}$ piston-cylinder diamond anvil cell, 8.75-mmdiameter and 12.5-mm-height. ${ }^{12}$ The diamond anvil cell was suspended from one of two concentric tubes. The internal tube acted as a ram so that the pressure could be modified by a drive mechanism at room temperature while the sample remained at $\mathrm{T}=4 \mathrm{~K}$. This probe also allowed us to use liquid helium as a pressure medium to assure a purely hydrostatic pressure. The preliminary 
4-K $2 \%$ nitrogen InGaAsN measurements reported in Refs. 7 and 8 were taken using methanol, ethanol, and water in a ratio of 16:3:1 for the pressure medium. We repeated the bandgap energy and linewidth measurements for the $2 \%$ nitrogen InGaAsN samples using the hydrostatic helium. pressure cell and found that the results from the helium and methanol/ethanol/water pressure cells were nearly identical, and hence in this paper, no distinction between these two separate results will be made.

The substrate upon which the sample was grown was mechanically lapped to a $1 / 2-\mu \mathrm{m}$-finish and thickness of $27 \mu \mathrm{m}$ and then chemically etched with a $3 \%$ solution of bromine in ethanol to a final thickness of $23 \mu \mathrm{m}$. The shift in the fluorescence of a small chip of ruby placed in the pressurized volume was used to calibrate the pressure at $4 \mathrm{~K}$ with an accuracy of $\pm 0.5 \mathrm{kbar} .{ }^{13} \mathrm{~A}$ single $600-\mu \mathrm{m}$-diameter optical fiber, butted up against one of the diamonds, directed the nominally 1 $\mathrm{mW}$ power 514.5-nm-wavelength laser to the sample and also collected the PL signal from the sample. A beam splitter system was used to direct the PL signal to an optical monochromator. Depending on the bandgap energy, two liquid-nitrogen-cooled detectors were used to detect the PL signal. For the long wavelength regimes, where the bandgap energies were near or below an energy of $1 \mathrm{eV}$, a NORTH-COAST EO-817L Ge-detector was employed, while for larger bandgap energies, a standard CCD array was used.

Two typical ambient pressure, low-temperature $(\mathrm{T}=4 \mathrm{~K})$ PL spectra for nominally $2 \%$ (left trace) and $1 \% \mathrm{~N}$ (right trace) in InGaAsN lattice matched to GaAs are shown in Fig. 1. As can be seen, the 4-K bandgap energies are nearly 1150 and $1225 \mathrm{meV}$, which are significantly less than the 4-K GaAs bandgap energy $E_{\mathrm{g}} \approx 1515 \mathrm{meV}$. The FWHM PL linewidths $\sigma(\mathrm{x})$ are about 14 and $22 \mathrm{meV}$ respectively for the nominally $1 \%$ and $2 \%$ nitrogen samples.

\section{Discussion}

Before we discuss how the variation of the exciton reduced-mass with pressure is determined from the study of the behavior of $\sigma$ with pressure, we briefly outline the basic ideas underlying the theory of excitonic linewidth in alloys. Excitonic transitions in semiconductor alloys as observed in optical measurements such as PL, PL excitation, and modulated reflectance are considerably broader than those observed in their binary components. The broadening mechanism has been attributed to compositional disorder which is inevitably present in these kinds of systems. In high 
quality alloys, this disorder is expected to be completely random. The physical origin of the excitonic line broadening due to compositional disorder lies in the fact that the average alloy composition inside the volume occupied by the exciton is different from that inside the volume of another exciton in a different spatial region of the alloy. Even though the global or average value of the alloy composition is fixed, excitons in different spatial regions in the alloy experience different local average values of the alloy composition. Within the framework of the virtual crystal approximation (VCA), the conduction and valence-band edges sampled by the exciton are determined by the local alloy composition. Therefore, excitons in different spatial regions have different optical transition energies, thus leading to inhomogeneous broadening of the transition energy. Here we implicitly assume that the exciton localization is entirely a result of the bandgap energy variations due to the random alloy fluctuations. Because of the large values of the variation of bandgap energy with composition (see below), motional averaging and other linewidth effects due to the thermal motion of the free exciton, as discussed in detail by Lyo ${ }^{12}$ are neglected. Furthermore, because the bandgap offset between $\mathrm{GaAsN}$ and GaAs for the 1 and $2 \%$ nitrogen alloys is small ${ }^{7}$ the contributions to the alloy fluctuation theory of line broadening by fluctuating valence-band hole energies ${ }^{14}$ is also ignored.

Based on this concept, several groups (see Lee and Bajaj $^{9}$ and references therein) have calculated the variation of the excitonic photoluminescence linewidth $\sigma(x)$ as a function of alloy composition. They essentially calculate the probability of an exciton with volume $V_{\text {exc }}$ experiencing an alloy composition $\mathrm{x}_{\mathrm{exc}}$ about the mean value of $\mathrm{x}$ and relate it to the transition energy using the VCA. In this model, the exciton PL lineshape function $f(E, x)$ is Gaussian, i.e.,

$$
f(E, x)=\frac{1}{\sqrt{2 \pi \sigma_{0}(x)}} \exp \left(-\frac{1}{2} \frac{\left(E-E_{g}(x)\right)^{2}}{\sigma_{0}(x)^{2}}\right)
$$

where $E_{g}(x)$ is the average bandgap energy for the alloy with composition $x$. From the alloy fluctuation model $^{9}, \sigma_{0}$ can be expressed as

$$
\sigma_{0}(x)^{2}=K^{2}\left(\frac{x(1-x) V}{\frac{4 \pi}{3} r_{e x c}^{3}}\right)\left(\frac{\partial E_{g}}{\partial x}\right)^{2}
$$


In this expression $V$ is the smallest volume over which the composition fluctuation can occur and for the zincblende structure is equal to $\left(a^{3} / 4\right), a$ being the lattice constant; the volume of the exciton is $(4 \pi / 3) r_{e x c}^{3}$, where the exciton Bohr radius is given by $r_{e x c}=\left(\hbar^{2} \kappa / \mu \mathrm{e}^{2}\right), \kappa$ is the dielectric constant, $\mathrm{e}$ is the electronic charge, and $\mu$ is the reduced mass of the exciton. The last term in Eq. (2), $\partial \mathrm{E}_{\mathrm{g}} / \partial \mathrm{x}$ is the variation of $\mathrm{E}_{\mathrm{g}}$ with alloy composition $\mathrm{x}$. Depending on the exact definition of the exciton volume, the value of $\mathrm{K}$ in Eq. (2) varies between 0.32 and 1 . For the analysis of the linewidth data reported here, we use $\mathrm{K}=0.4$. This value of $\mathrm{K}$ has been derived by Zimmerman ${ }^{15}$ and independently by Lee and Bajaj ${ }^{9}$ using somewhat similar quantum mechanical approaches which do not involve explicitly an ill defined concept of the so called exciton volume. In terms of $\sigma_{0}(\mathrm{x})$ and the Gaussian lineshape $\mathrm{f}(\mathrm{E}, \mathrm{x})$, Eq. (1), the FWHM linewidth $\sigma(\mathrm{x})$ is

$$
\sigma(x)=2 \sqrt{2 \ln 2} \sigma_{0}(x)
$$

Thus from Eqs. (2) and (3), depending on the requirement, the exciton FWHM linewidth $\sigma$ can be described in terms of the reduced mass $\mu$ or conversely, the reduced exciton mass $\mu$ can be described in terms of the FWHM $\sigma(\mathrm{x})$, i.e., $\sigma \propto \mu^{3 / 2}$ or $\mu \propto \sigma^{2 / 3}$. Using GaAs values for the dielectric constant $\kappa=12.5$ and for the lattice constant $a=5.6 \times 10^{-8} \mathrm{~cm}$, an expression for $\mu$ can be written in terms of the FWHM $\sigma(\mathrm{x}), \mathrm{x}$, and $\partial \mathrm{E}_{\mathrm{g}} / \partial \mathrm{x}$ as

$$
\mu=\left(\frac{5.3 \sigma(x)}{\sqrt{x(1-x)} \frac{\partial E_{g}}{\partial x}}\right)^{2 / 3}
$$

Traditionally, models for the linewidths $\sigma(x)$ are calculated in terms of the exciton reduced mass $\mu$ and then compared with the experimental values (See for example Lee and Bajaj ${ }^{9}$ and references therein.) As mentioned above, the differences between the various theoretical treatments concerned the definition of the exciton volume. In this paper, we accept the validity of the alloy fluctuation model for the linewidth (Eqs. (2) and (3)) and that we use the experimental linewidth to infer the mass. From the PL spectra shown in Fig. 1 and with $\mathrm{K}=0.4$ and $\partial \mathrm{E}_{\mathrm{g}} / \partial \mathrm{x} \approx 20 \mathrm{eV},{ }^{8}$ the exciton reduced mass for the $1 \%$ sample can be calculated from Eq. (4) with the result $\mu \sim 0.10$ 
for the $1 \%$ nitrogen structure, and for the $2 \%$ nitrogen sample, $\mu \sim 0.13$. These mass values $\sim 0.13$ for the $2 \%$ nitrogen in InGaAsN agree with the aforementioned experimental results obtained by the more conventional optical determinations. ${ }^{7}$ This agreement between the calculated mass from the FWHM linewidth data and other mass determinations is thus the basis of this paper for using the pressure dependence of $\sigma(x)$ to obtain pressure dependent masses.

The 4-K pressure dependencies of the bandgap energy for the nominally $1 \%$ nitrogen (open circles) and 2\% nitrogen (open squares) InGaAsN samples are shown in Fig. 2. Some of the data for the $2 \%$ nitrogen structure is taken from Refs. 7 and 8 and some were taken with the helium pressure cell. The smooth curves drawn through the data are meant to be an aid to the eye. As can be seen from Fig 2, the energy separation between the $1 \%$ and $2 \%$ nitrogen alloys bandgap energies is less at high pressures than at ambient pressure. Similar results have been reported by Shan, et. al. ${ }^{16}$ who measured the pressure dependence of the photomodulated reflectivity signal for various nitrogen concentrations in InGaAsN and GaAsN alloys. Thus, because of the repulsion between the $\Gamma$-bands and $X$-bands at high pressures, the difference between bandgap energies for differing nitrogen concentrations tend to diminish. ${ }^{7,16}$ Thus if the alloy fluctuation theory for the pressure dependence for FWHM linewidth is going to be used in order to obtain mass data, the pressure dependence of $\partial \mathrm{E}_{\mathrm{g}} / \partial \mathrm{x}$ must be considered. The pressure dependence $\partial \mathrm{E}_{\mathrm{g}} / \partial \mathrm{x}(\mathrm{P})$ can be extracted from the data shown in Fig. 2 or from the data reported in Ref. 16. The right hand axis of Fig. 2 shows the experimentally determined pressure dependence for $\partial \mathrm{E}_{\mathrm{g}} / \partial \mathrm{x}$ from the data from Shan et. al. ${ }^{16}$ The experimental curve for $\partial \mathrm{E}_{\mathrm{g}} / \partial \mathrm{x}$ is obviously very sensitive to the accuracy of the knowledge of the nitrogen concentration for InGaAsN alloys. This kind of data for established and extensively studied alloy systems such as $\mathrm{Al}_{\mathrm{x}} \mathrm{Ga}_{1-\mathrm{x}} \mathrm{As}$ alloys have been well documented in the literature. ${ }^{17}$ However, for InGaAsN alloys, the "bowing parameters" for $\partial \mathrm{E}_{\mathrm{g}} / \partial \mathrm{x}$ and also the pressure dependence are still being determined. ${ }^{18-20}$ The main reason for this problem is that an accurate determination of alloy compositions for quarternary alloy systems such as $\operatorname{In}_{\mathrm{y}} \mathrm{Ga}_{1-\mathrm{y}} \mathrm{As}_{1-}$ ${ }_{x} N_{x}$ at low indium and nitrogen concentrations $x$ and $y$ is difficult. Thus for purposes of demonstrating the technique of using the pressure dependence of the FWHM linewidth to determine mass measurements, we will use the data of Shan et. al., ${ }^{16}$ to derive the pressure dependence for $\partial \mathrm{E}_{\mathrm{g}} / \partial \mathrm{x}(\mathrm{P})$ and is presented in Fig. 2. 
The pressure dependence of the FWHM linewidth for the $1 \%$ (solid circles) and $2 \%$ nitrogen (open squares) InGaAsN samples is shown in Fig. 3. The smooth curves are drawn through both sets of data are an aid to the eye. As can be seen in the figure, the FWHM linewidths increase dramatically with pressure changing from about an ambient pressure value of $20 \mathrm{meV}$ to greater than $100 \mathrm{meV}$ for $100 \mathrm{kbars}$.

From Eq. (4), using the FWHM pressure data shown in Fig. 3, the $\partial \mathrm{E}_{\mathrm{g}} / \partial \mathrm{x}(\mathrm{P})$ curve shown in Fig., 2, we calculate the pressure dependence for exciton reduced mass $\mu$ with the result shown in Fig. 4. The solid circles are for $1 \%$ nitrogen values, the open squares are the $2 \%$ nitrogen values, and the open triangles are from the LDA calculation discussed below. The smooth curve is drawn through the $1 \%$ nitrogen data. We find that the values of the reduced exciton mass $\mu$ increase with pressure till about $100 \mathrm{kbars}$ and then tend to saturate and also that the pressure dependence of the 1 and $2 \%$ nitrogen samples are similar. The pressure coefficients are non-linear and large, especially when compared to that found in well behaved GaAs-type alloys. ${ }^{21,22}$

Because of our success in using the results of a first-principles electronic band structure calculation ${ }^{7}$ to quantify the change in the bandgap energy with pressure, we performed preliminary LDA calculations for the pressure dependence of the conduction-band mass. Our results are preliminary and hence will not be discussed in detail here. We can, however, make some qualitative statements by examining the LDA results reported in Ref. 7. As discussed in that paper, the band repulsion between the $\Gamma$-like and $\mathrm{X}$-like bands at high pressure indicate that strong $\Gamma-\mathrm{X}$ mixing is occurring. The amount of $\Gamma-\mathrm{L}$ and $\Gamma-\mathrm{X}$ mixing is pressure dependent with the contribution from the $\Gamma$ - $\mathrm{L}$ mixing decreasing with pressure and while that from the $\Gamma-\mathrm{X}$ amount is increasing with pressure. Because the mass of the six-fold degenerate $\mathrm{X}$-point in $\mathrm{GaAs}$ is heavy $\left(\mathrm{m}_{\mathrm{Xt}}=1.2\right.$ and $m_{X I}=0.27$ ), we expect that the $\Gamma-X$ mixing will cause a corresponding pressure dependent increase to the $\Gamma$-like conduction-band mass by the heavy X-like mass. In order to either (1) extract the pressure dependent conduction-band mass from the reduced exciton mass or (2) calculate the pressure dependence of the exciton mass, knowledge of the pressure dependence of the valence-band mass is necessary. Experimentally, the change to the valence-band mass is about $1 \% / \mathrm{kbar}$ for well behaved GaAs-type alloys. ${ }^{21}$ To this end, we have also calculated the pressure dependence of the GaAs valence-band mass using our LDA program. The calculated change in 
the exciton reduced mass with pressure is displayed in Fig. 3 (solid triangles) and shows enhancement with pressure similar to that observed experimentally. An improved calculation of the conduction band mass requires information about the valence-band states, the $\mathrm{X}$-like as well as the $\mathrm{L}$ like masses. In the future, an obvious goal of our LDA calculations will be to predict the dependence shown in Fig. 3.

It should be pointed out that the near-bandedge emission we observe is most likely associated with excitons bound by the local alloy fluctuations in which case the excitonic wave function is only slightly perturbed by the alloy fluctuations. Therefore the theory of excitonic broadening due to alloy fluctuations which depends on free exciton wavefunctions is still applicable. Also the values of the exciton reduced mass we derive from excitonic linewidth represents an upper limit as we have not taken into account other broadening mechanisms

\section{Conclusions}

We report the variation of the excitonic linewidth $\sigma$ as a function of pressure up to 120 kbars in InGaAsN with $7 \%$ indium and $1 \%$ nitrogen using photoluminescence spectroscopy at $4 \mathrm{~K}$. We find that the value of the excitonic linewidth increases as a function of pressure until about $100 \mathrm{kbars}$ and then tends to saturate. The reduced mass of the exciton as a function of pressure is derived from the observed variation of the excitonic linewidth using a theoretical formalism which is based on the broadening of the excitonic transition is caused by the potential fluctuations in the alloy. The variation of the reduced mass with pressure thus determined is examined in view of the results of a recent first-principles band structure calculation using local density approximation.

\section{Acknowledgements}

The authors wish to thank Dr. Wei Shan for providing his data for $\partial \mathrm{E}_{\mathrm{g}} / \partial \mathrm{x}$ shown Fig. 2. Sandia is a multiprogram laboratory operated by Sandia Corporation, a Lockheed Martin Company, for the United States Department of Energy under contract DE-AC04-94AL85000. Part of this work was performed at the National High_Magnetic Field Laboratory, which is supported by NSF Cooperative Agreement No. DMR-9016241 and by the State of Florida. 


\section{References}

1. M. Weyers, M. Sato, and H. Ando, "Red shift of photoluminescence and absorption in dilute GaAsN alloy layers," Jpn. J. Appl. Phys, 31, Pt. 2 pp. L853-L855 (1992).

2. M. Weyers and M. Sato, "Growth of GaAsN alloys by low-pressure metalorganic chenmical vapor-deposition using plasma-cracked $\mathrm{NH}_{3}$," Appl. Phys. Lett. 62, pp. 1396-1398 (1993).

3. M. Kondow, K. Uomi, A. Niwa, T. Kitatani, S. Watahiki, and Y. Yazawa, "GaInNAs: A novel material for long-wavelength-range laser diodes with excellent high-temperature performance," Jpn. J. Appl. Phys. 35, pp. 1273-1275 (1996).

4. M. Kondow, T. Kitatani, S. Nakatsuka, M. C. Larson, K. Nakahara, Y. Yazawa, M. Okai, and K. Uomi, "GaInNAs: A novel material for long wavelength semiconductor lasers," IEEE J. Selected Topics in Quantum Electronics 3, pp. 719-729 (1997).

5. T. Miyamoto, K. Takeuchi, F. Koyama, and K. Iga, "A novel GaInNAs-GaAs quantum-well structure for long-wavelength semiconductor lasers," IEEE Photonics Tech. Lett. 9, pp. 14481450 (1997).

6. E. D. Jones, A. A. Allerman, D. J. Friedman, F, F, Geisz, J. F. Klem, S. R. Kurtz, N. R. Modine, W. Shan, and W. Walukiewicz, "Next generation thin films for photovoltaics: InGaAsN" in Photovoltaics for the 2 Ist Century, Edited by V. A. Kapur, R. D. McConnell, D. Carlson, G. P. Ceasar, and A. Rohatgi (Electrochemical Society, Pennington, NJ 1999), pp. 252-267.

7. E. D. Jones, N. A. Modine, A. A. Allerman, S. R. Kurtz, A.F. Wright, S. T. Tozer, and X. Wei, "Band structure of InGaAsN alloys and effects of pressure," Phys. Rev. B60, pp. 4430-4433 (1999).

8. E. D. Jones, N. A. Modine, A. A. Allerman, I. J. Fritz, S. R. Kurtz, A. F. Wright, S. T. Tozer, and X. Wei, "Optical properties of InGaAsN: A new leV bandgap material system," in LightEmitting Diodes: Research, Manufacturing, and Applications III, edited by E. F. Schubert, I. T. Ferguson, and H. W Yao, SPIE Conference Proceedings Vol. 3621 (International Society for Optical Engineering, Bellingham, WA, 1999), pp. 52-63.

9. S. M. Lee and K. K. Bajaj, "A quantum statistical theory of linewidths of radiative transitions due to compositional disordering in semiconductor alloys" J. Appl. Phys. 73, pp. 1788-1796 (1993).

10. A. A. Allerman, S. R. Kurtz, E. D. Jones, J. M. Gee, J. C. Banks, and A. Clement-font, "The growth of InGaAsN for high efficiency solar cells by metalorganic chemical vapor deposition" in Photovoltaics for the 21st Century, Edited by V. A. Kapur, R. D. McConnell, D. Carlson, G. P. Ceasar, and A. Rohatgi (Electrochemical Society, Pennington, NJ 1999), pp. 178-184.

11. D. M. Follstaedt (Unpublished results)

12: S.W. Tozer (Unpublished results) 
13. R. A. Forman, G. J. Piermarini, J. D. Barnett, S. Block, "Pressure measurement made by the utilization of ruby sharp-line luminescence," Science 176, pp. 284-285 (1972).

14. S. K. Lyo, "Theory of magnetic-field-dependent alloy broadening of exciton-photoluminescence linewidths in semiconductor alloys" Phys. Rev. B38, pp. 2152-2161 (1993).

15. R. Zimmerman, "Theory of exciton linewidth in II-VI semiconductor mixed crystals" J. Crystal Growth 101, pp. 346-349 (1990)

16. W. Shan, W. Walukiewicz, and J. W. Ager III, "Band anticrossing in GaInNAs alloys," Phys. Rev Lett. 82, pp. 3312-2215 (1999).

17. S. Adachi, Physical Properties of III-V Semiconductor Compounds (John Wiley \& Son, NY, 1992).

18. W. G. Bi and C. W. Tu, "Bowing parameter of the band-gap energy of $\mathrm{GaN}_{\mathbf{x}} \mathrm{As}_{1-\mathrm{x}}$," Appl. Phys. Lett. 70, pp. 1608-1610 (1997).

19. M. Kondow, T. Kitatani, M. C. Larson, K. Nakahara, and K. Uomi, "Gas source MBE of GaInNAs for long-wavelength laser diodes," presented at the 6th Int. Conf. On CBE and Related Techniques, Montreux, Switzerland, 1997

20. L. Malikova, F. H. Pollak, and R. Bhat, "Composition and temperature dependence of the direct band gap of $\mathrm{GaAs}_{1-\mathrm{x}} \mathrm{N}_{\mathrm{x}}(0 \leq \mathrm{x} \leq 0.0232)$ using contactless electroreflectance," J. Electronic Materials 27, pp. 484-487 (1998)

21. .E. D. Jones, H. Ackermann, J. E. Schirber, T. J. Drummond, L. R. Dawson, and I. J. Fritz, "Magneto-optic determinations of the pressure-dependent band-gap energies and effective masses in strained-layer superlattices," Appl. Phys. Lett. 47, pp. $492-494$ (1985).

22. E. D. Jones, S. T. Tozer, and T. Schmiedel, "Pressure Dependence of the Bandgap Energy and the Conduction-Band Mass for an n-type InGaAs/GaAs Single-Strained Quantum Well," Physica E 2, pp. 146-150 (1998). 


\section{Figure Captions}

Figure 1. Ambient pressure PL spectra at $4 \mathrm{~K}$ and for $1 \%$ (right trace) and $2 \%$ (left trace) nitrogen in InGaAsN. The FWHM linewidths are $\sigma(1 \% \mathrm{~N}) \sim 14 \mathrm{meV}$ and $\sigma(2 \% \mathrm{~N}) \sim 22 \mathrm{meV}$.

Figure 2. The pressure dependencies of the bandgap energy for the $1 \%$ nitrogen (open circles) and $2 \%$ nitrogen (open squares) InGaAsN samples for $\mathrm{T}=4 \mathrm{~K}$. The data for the $2 \%$ nitrogen sample shown is taken from Refs. 7 and 8 and data taken here with the helium pressure cell. The smooth curves drawn through the data are an aid to the eye. The right-hand axis shows the pressure variation of $\partial \mathrm{E}_{\mathrm{g}} / \partial \mathrm{x}$ from Ref. 16.

Figure 3. The pressure dependence of the FWHM for the $1 \%$ (closed circles) and $2 \%$ (open squares) at $\mathrm{T}=4 \mathrm{~K}$. The smooth curves drawn through the data are an aid to the eye.

Figure 4. Exciton reduced effective masses $\mu$ versus pressure for $1 \%$ nitrogen (solid circles) calculated from Eq. (4) using the 4-K FWHM data shown in Fig. 3 and the $\partial \mathrm{E}_{\mathrm{g}} / \partial \mathrm{x}$ curve shown in Fig. 2. The open squares are for $2 \%$ nitrogen and are based on data reported in Refs. 7 and 8 and new data taken with the helium pressure cell. The smooth curve drawn through the $1 \%$ nitrogen mass data is meant to be an aid to the eye. The open triangles are the pressure dependence LDA calculated reduced mass. 


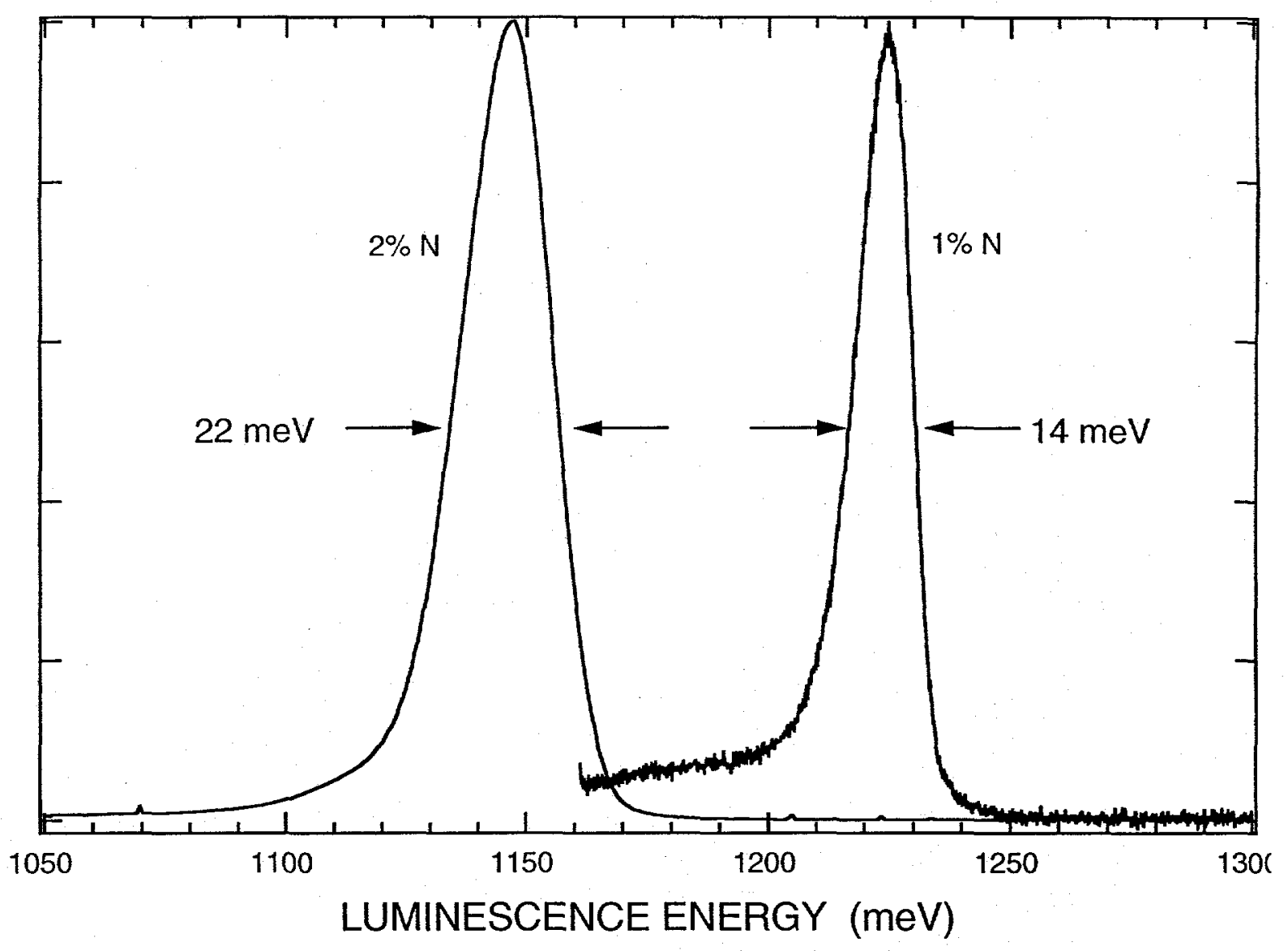




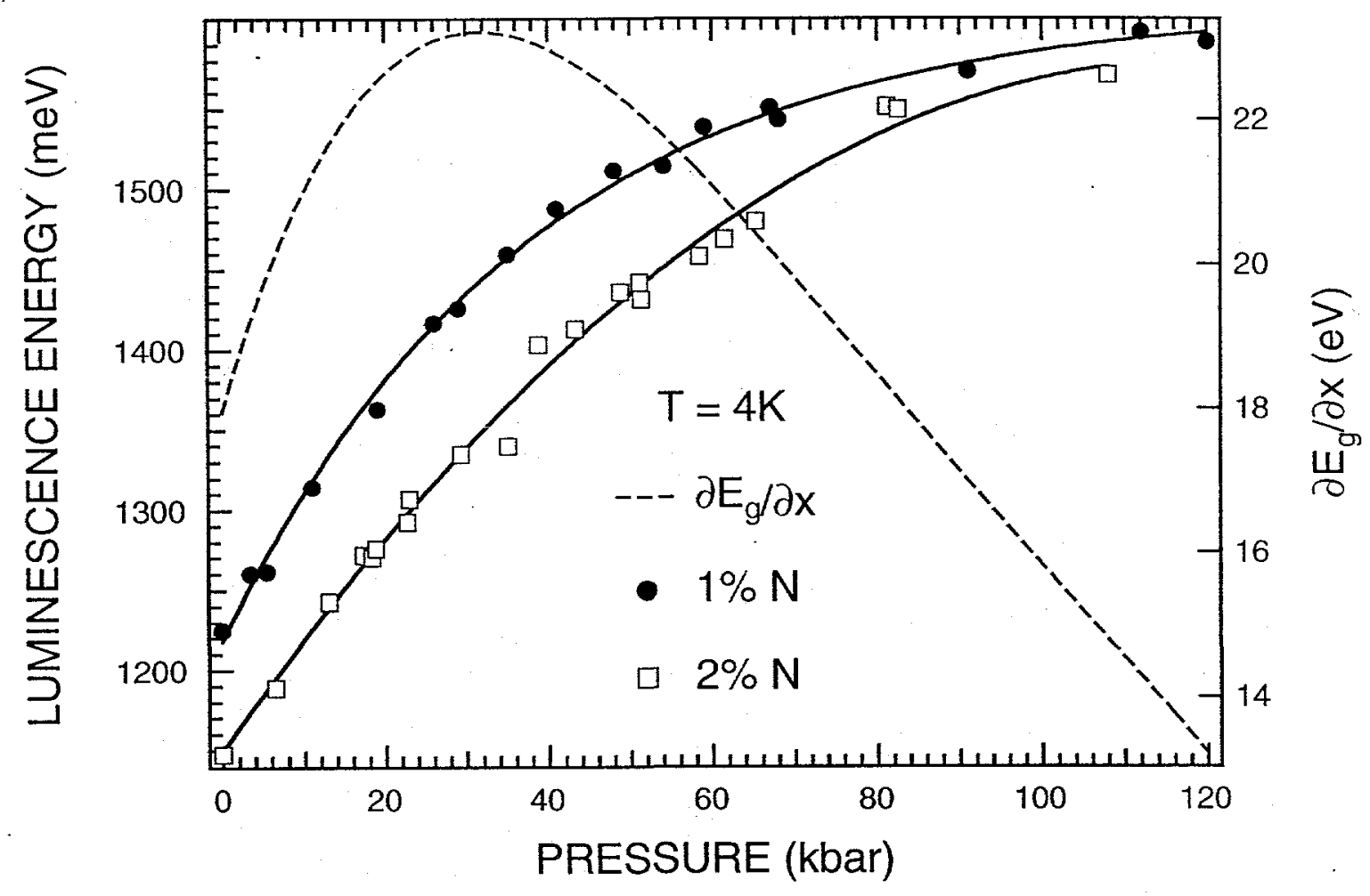




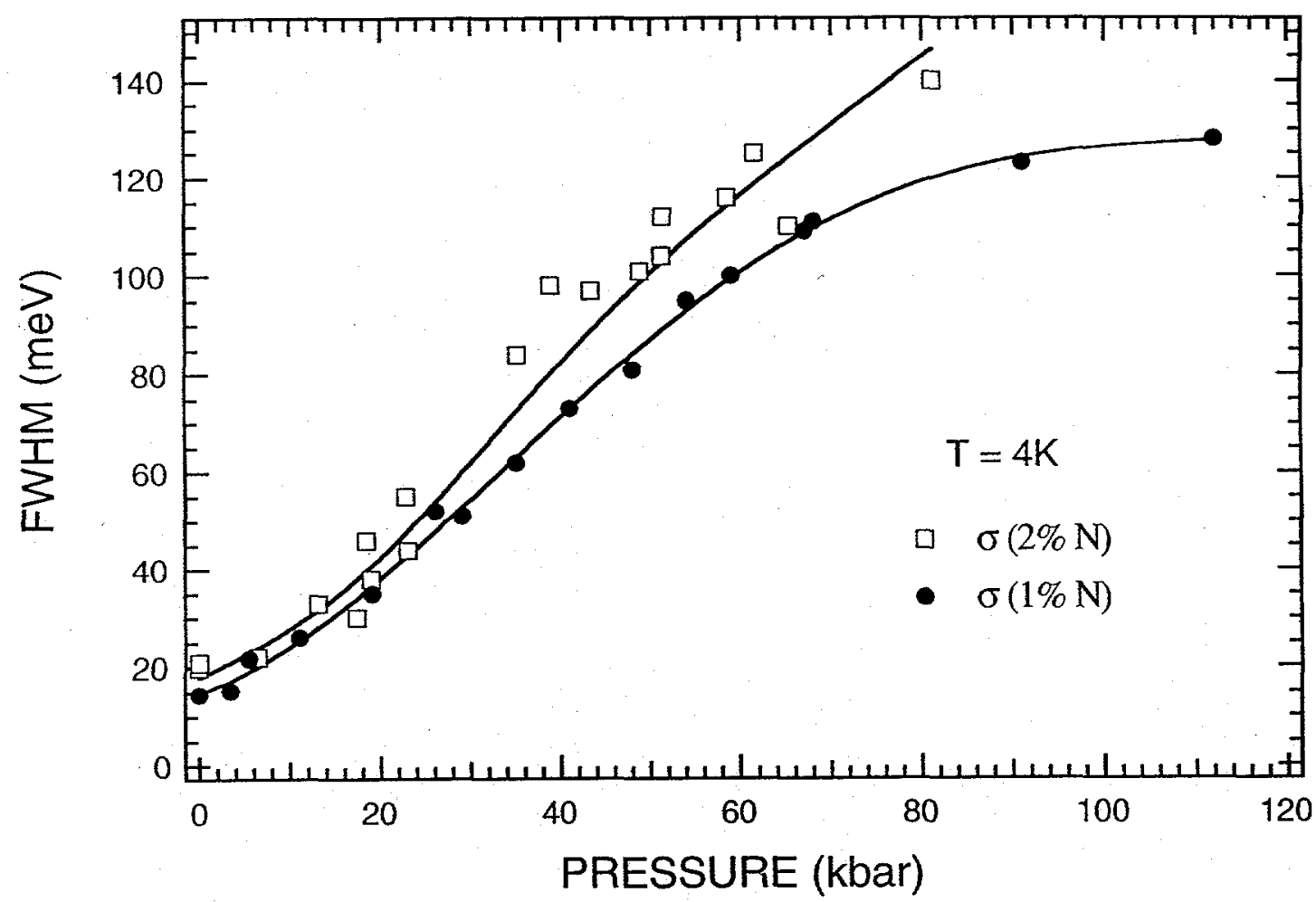




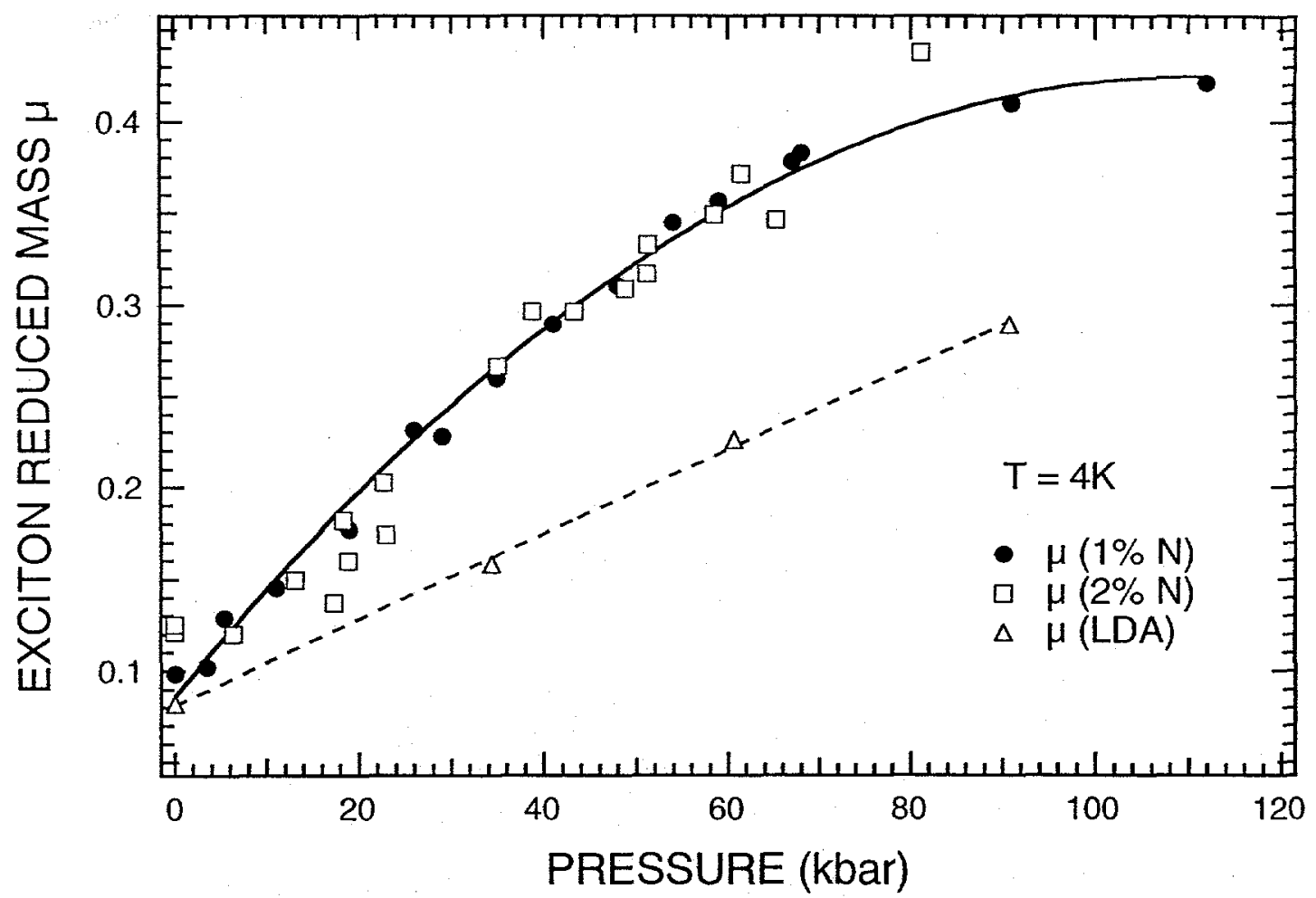

